\title{
Path Integral Formulation for Ionic Broadening in Plasmas: Lyman- $\alpha$ with Fine Structure and Dynamical Effects
}

\author{
N. Bedida, M. T. Meftah \\ Laboratoire de Rayonnement et Plasmas et Physique des Surfaces (LRPPS), Université Kasdi Merbah Ouargla, \\ Ouargla, Algérie \\ Email: n_bedida@yahoo.fr,mewalid@yahoo.com
}

Received August 19, 2012; revised September 17, 2012; accepted September 24, 2012

\begin{abstract}
Using the path integral formalism, the fine structure and dynamics effects are taken into account for the broadening of spectral lines in a plasma. A compact expression of the dipolar autocorrelation function for an emitter in the plasma is derived for Lyman alpha lines with fine structure. The expression of the dipolar autocorrelation function takes into account the dynamics effects, which are represented by the time microfield autocorrelation function.
\end{abstract}

Keywords: Path Integrals; Autocorrelation Function; Electric Dipole; Fine Structure; Dynamical Effects

\section{Introduction}

The spectral line shapes of radiative atoms and ions in the plasma provide valuable diagnostic tools for a number of physical quantities, such as the density and temperature of charged particles, the transported radiative energy, and possibly the determination of electric fields [1]. The shape of lines in a plasma results from the interactions between the radiator and all constituents (neutrals, electrons and ions) of the plasma. With variable contributions depending on plasma conditions, causes of broadening are the Doppler effect, which is produced by the movement of the radiator, natural broadening, due to the finite lifetime of the atomic excited state, and what will be the focus of this paper, the Stark broadening which is due to the interaction between the radiator and the electric field of the two kind of perturbers (ions-electrons) [2]. This problem has been widely studied using the standard Hamiltonian approach of quantum mechanics. It started with the work of Baranger [3], and Kolb and Griem [4]. In these classic papers on Stark broadening, the electrons are treated within the impact theory, and the ions in the quasi-static approximation. Both kind of particles having a Coulomb interaction with the radiator, the difference between ions and electrons is merely due to their velocity difference. For many plasma conditions, ions are slow enough to justify the use of a quasi-static approximation, but for hydrogen plasmas. In our investigation, we introduce an alternative method able to take into account the fine structure and the dynamics effects. This method is based on the Feynman path integral formalism [5,6] which deals with electrons and ions on the same physical basis. The general frame for this formalism has been previously developped $[7,8]$, but has then only be applied to the static ion case. Using this formalism, one can treat time-independent and time-dependent problems on the same footing, which is a real advantage over the standard Hamiltonian approach when solving time-dependent problems.

In this paper we retrieved the formula of the dipolar auto-correlation function common in the line broadening theory. Our derivation uses the Feynman path integral formalism. Since the mean time of the electron-emitter collision is negligible compared with the ion-emitter one, we shall replace the electron-emitter collision effects by a standard collision operator, whereas the ion-emitter collisions effects, via the dipole approximation, will be treated in the perturbative approach using the path integral formalism. Section 2 is concerned by with rather low density, and/or high temperature, this static approximation may however no longer be valid. The formulation of the dipolar auto-correlation function and in Section 3, we apply earlier results to the Lyman alpha line with fine structure in time-dependent electric microfield. Conclusion and perspectives are given in Section 4.

\section{The Spectral Line Shape in the Path Integrals Theory}

We start here by the time dipolar autocorrelation function 
of the radiator from which the spectral line shapes are generally deduced. The emitter is perturbed by ions and electrons treated as charged particles moving on classical paths. For a description of the radiator perturber interaction, it is usually sufficient to keep only the first term in the multipole expansion, using the so-called dipolar approximation. As quoted before, the effect of the electrons is usually treated with the impact theory by a collision operator. Our path integral approach could be applied to both electrons and ions. The electric microfield appearing in our formalism could thus be created by the electrons, the ions or both kind of particles. The usual start of spectral line shape theory is the general formula giving the radiation power [9]:

$$
I(\omega)=\frac{1}{\pi} \operatorname{Re} \int_{0}^{\infty} C(t) \mathrm{e}^{-\mathrm{i} \omega t} \mathrm{~d} t
$$

where $C(t)$ is the auto-correlation function of the dipolar momentum of the emitter given by:

$$
C(t)=\sum_{\alpha \alpha^{\prime} \beta \beta^{\prime}} \mathbf{d}_{\alpha \beta}\left\{\beta\left|T_{b}(t, 0)\right| \beta^{\prime} \alpha\left|T_{a}(t, 0)\right| \alpha^{\prime}\right\} \mathbf{d}_{\alpha^{\prime} \beta^{\prime}}^{*}
$$

where $\alpha \alpha^{\prime}$ and $\beta \beta^{\prime}$ are the upper and lower states respectively of the emitter including the spin states, and $\{\cdots\}$ stands for a statistical average over the perturbers.
As we are concerned in this work by the Lyman structure, we have in this case that the lower state $\beta, \beta^{\prime}$ is degenerate, and we can, after using the representation $\left|n, j, m_{j}\right\rangle$, transform $C(t)$ as:

$$
\begin{aligned}
& C(t)=\mathrm{e}^{-\frac{\mathrm{i} \epsilon \epsilon_{1}}{\hbar}} \sum_{j j^{\prime} m_{j} m_{j^{\prime}}} \\
& {\left[\sum_{m}\left\langle n j m_{j}|\boldsymbol{d}| 1 \frac{1}{2} m\right\rangle\left\langle n j^{\prime} m_{j^{\prime}}|\boldsymbol{d}| 1 \frac{1}{2} m^{*}\right\rangle \cdot\left\{n j m_{j}\left|T_{a}\right| n j^{\prime} m_{j^{\prime}}\right\}\right]}
\end{aligned}
$$

Using the Wigner-Eckart theorem, we obtain in the $\left|n, j, m_{j}\right\rangle$ representation:

$$
C(t)=\sum_{j m_{j}} \frac{\mathrm{e}^{-\frac{\mathrm{i} t \epsilon_{1}}{\hbar \frac{1}{2}}}}{2 j+1}|n j| \mathrm{d}^{1}\left|1 \frac{1}{2}\right|^{2}\left\{n j m_{j}\left|T_{a}\right| n j^{\prime} m_{j^{\prime}}\right\}
$$

where $\mathrm{d}^{1}$ is the reduced matrix element, $\epsilon_{1}$ the energy corresponding to $(n=1, j=1 / 2)$ and the matrix element of the evolution operator $T$ in upper state $a$.

\section{Application to Lyman- $\alpha$ Broadening}

In the case of Lyman alpha line with fine structure, the autocorrelation function can be written as:

$$
\begin{aligned}
C(t) & =\mathrm{e}^{-\frac{\mathrm{i} t \epsilon_{1}}{\hbar}} \sum_{j m_{j}}\left[\frac{1}{4}\left|2 \frac{3}{2}\right| \mathrm{d}^{1}\left|1 \frac{1}{2}\right|^{2} \mathrm{e}^{\Phi_{e}^{1} t} \sum_{m_{j}=-\frac{3}{2}}^{+\frac{3}{2}}\left\{2 \frac{3}{2} m_{j}\left|T_{a}\right| 2 \frac{3}{2} m_{j}\right\}+\frac{1}{2}\left|2 \frac{1}{2}\right| \mathrm{d}^{1}\left|1 \frac{1}{2}\right|^{2} \mathrm{e}^{\Phi_{e}^{2} t} \sum_{m_{j}=-\frac{1}{2}}^{+\frac{1}{2}}\left\{2 \frac{1}{2} m_{j}\left|T_{a}\right| 2 \frac{1}{2} m_{j}\right\}\right] \\
& =\mathrm{e}^{-\frac{\mathrm{i} \epsilon \epsilon_{1} \frac{1}{2}}{\hbar}}\left[\frac{1}{4}\left|2 \frac{3}{2}\right| \mathrm{d}^{1}\left|1 \frac{1}{2}\right|^{2} \mathrm{e}^{\Phi_{e}^{1} t}\left(C_{1}(t)+C_{2}(t)+C_{3}(t)+C_{4}(t)\right)+\frac{1}{2}\left|2 \frac{1}{2}\right| \mathrm{d}^{1}\left|1 \frac{1}{2}\right|^{2} \mathrm{e}^{\Phi_{e}^{2} t}\left(C_{5}(t)+C_{6}(t)\right)\right]
\end{aligned}
$$

where

$$
\begin{aligned}
& C_{1}(t)=\left\{2 \frac{3}{2} \frac{3}{2}\left|T_{a}\right| 2 \frac{3}{2} \frac{3}{2}\right\} C_{2}(t)=\left\{2 \frac{3}{2} \frac{1}{2}\left|T_{a}\right| 2 \frac{3}{2} \frac{1}{2}\right\} C_{3}(t)=\left\{2 \frac{3}{2} \frac{-1}{2}\left|T_{a}\right| 2 \frac{3}{2} \frac{-1}{2}\right\} \\
& C_{4}(t)=\left\{2 \frac{3}{2} \frac{-3}{2}\left|T_{a}\right| 2 \frac{3}{2} \frac{-3}{2}\right\} C_{5}(t)=\left\{2 \frac{1}{2} \frac{1}{2}\left|T_{a}\right| 2 \frac{1}{2} \frac{1}{2}\right\} C_{6}(t)=\left\{2 \frac{1}{2} \frac{-1}{2}\left|T_{a}\right| 2 \frac{1}{2} \frac{-1}{2}\right\}
\end{aligned}
$$

and $\Phi_{\mathrm{e}}^{\mathrm{i}=1,2}$ are the collision operators.

The matrix element of the evolution operator $T$ in the upper state $a$ is:

$$
\alpha\left|T_{q}(t, 0)\right| \alpha^{\prime}=\int \mathrm{d} \boldsymbol{r} \mathrm{d} \boldsymbol{r}^{\prime} \phi_{\alpha}^{*}(\boldsymbol{r}) K_{a}\left(\boldsymbol{r}^{\prime}, t / \boldsymbol{r}, 0\right) \phi_{\alpha^{\prime}}\left(\boldsymbol{r}^{\prime}\right)
$$

where $K_{a}\left(\boldsymbol{r}^{\prime}, t / \boldsymbol{r}, 0\right)$ is the Feynman propagator describing the emitter evolution in the surrounding ion plasma. $\phi_{\alpha}(\boldsymbol{r})$ are the eigen functions of the Dirac operator relative to the free hydrogen atom. Then, to calculate the dipolar auto-correlation function $C(t)$ it is useful to evaluate the Feynman propagator $K_{a}\left(\boldsymbol{r}^{\prime}, t / \boldsymbol{r}, 0\right)$ as follows:

$$
K_{a}\left(\boldsymbol{r}^{\prime}, t / \boldsymbol{r}, 0\right)=\int_{\boldsymbol{r}(0)=\boldsymbol{r}}^{\boldsymbol{r}(t)=\boldsymbol{r}^{\prime}} D[\boldsymbol{r}(\tau)] \mathrm{e}^{-\mathrm{i}_{\hbar_{0}}^{t}\left(L_{0}^{D}+\mathrm{er} \cdot E(\tau)\right) \mathrm{d} \tau}
$$

where $L_{0}^{D}$ is the Dirac Lagrangian for the free hydrogen atom, and ( $\mathrm{r} \cdot \boldsymbol{E}(\tau)$ ) is the interaction between the hydrogen atom and the surrounding plasma in the dipole approximation.

It is possible to develop the propagator $K_{a}\left(\boldsymbol{r}^{\prime}, t / \boldsymbol{r}, 0\right)$ 
as a perturbation series knowing the free propagator $K_{0}$ relative to the free hydrogen atom:

$$
\begin{aligned}
K_{a}\left(r^{\prime}, t ; r, 0\right) \\
=\sum_{\alpha_{1} \cdots \alpha_{k+1}} \sum_{k=0}^{\infty}\left(\frac{\mathrm{i}}{k}\right)^{k} \frac{1}{k !} \int_{o}^{t} \mathrm{~d} \tau_{1} \cdots \int_{o}^{t} \mathrm{~d} \tau_{k} \int \mathrm{d} \boldsymbol{r} \cdots \int \mathrm{d} \boldsymbol{r}_{k}\left(\mathrm{e} \boldsymbol{E}_{1} \boldsymbol{r}_{1}\right) \cdots\left(\mathrm{e} \boldsymbol{E}_{k} \boldsymbol{r}_{k}\right) \cdots \phi_{\alpha_{k+1}}^{*}(\boldsymbol{r}) \phi_{\alpha_{k+1}}\left(\boldsymbol{r}_{k}\right) \\
\\
\cdot \exp \frac{\mathrm{i}}{\hbar} \epsilon_{\alpha_{k+1}}\left(t-\tau_{k}\right) \cdot \phi_{\alpha_{k}}(\boldsymbol{r}) \phi_{\alpha_{k-1}}^{*}\left(\boldsymbol{r}_{k-1}\right) \cdot \exp \frac{\mathrm{i}}{\hbar} \epsilon_{\alpha_{k}}\left(\tau_{k}-\tau_{k-1}\right) \cdots \phi_{\alpha_{2}}\left(\boldsymbol{r}_{2}\right) \phi_{\alpha_{2}}^{*}\left(\boldsymbol{r}_{1}\right) \\
\cdot \exp \frac{\mathrm{i}}{\hbar} \epsilon_{\alpha_{2}}\left(\tau_{2}-\tau_{1}\right) \phi_{\alpha_{1}}\left(\boldsymbol{r}_{1}\right) \phi_{\alpha_{1}}^{*}\left(\boldsymbol{r}^{\prime}\right) \cdot \exp \frac{\mathrm{i}}{\hbar} \epsilon_{\alpha_{1}}\left(\tau_{1}-0\right) .
\end{aligned}
$$

where $l=j \pm 1, l^{\prime}=2 j-l$ and $\epsilon_{n j}$ is the spectra of the where free atom including the fine structure.

The eigenfunctions of Dirac Hamiltonian in Coulombian field [10] are given by:

$$
\begin{gathered}
\phi_{n j}(\boldsymbol{r})=\left(\begin{array}{c}
f(\boldsymbol{r}) \Omega_{j l m_{j}} \\
(-1)^{\frac{1+l+l^{\prime}}{2}} g(\boldsymbol{r}) \Omega_{j l^{\prime} m_{j}}
\end{array}\right) \quad(10) \begin{array}{r}
\Omega_{j l^{\prime} m_{j}} \text { are the sph } \\
\text { radial functions and } \\
\text { Knowing that } \Omega_{j l} \\
\text { monics as: }
\end{array} \\
\Omega_{l+\frac{1}{2}, l, m_{j}}=\left(\begin{array}{l}
\sqrt{\frac{j+m_{j}}{2 j}} Y_{l}^{m_{j}-\frac{1}{2}} \\
\sqrt{\frac{j-m_{j}}{2 j}} Y_{l}^{m_{j}+\frac{1}{2}}
\end{array}\right) \Omega_{1-\frac{1}{2}, 1, \mathrm{~m}_{j}}=\left(\begin{array}{l}
-\sqrt{\frac{j-\mathrm{m}_{j}+1}{2 j}} \mathrm{Y}_{1}^{\mathrm{m}_{j}-\frac{1}{2}} \\
\sqrt{\frac{j+\mathrm{m}_{j}+1}{2 j}} \mathrm{Y}_{l}^{\mathrm{m}_{j}+\frac{1}{2}}
\end{array}\right)
\end{gathered}
$$

$\Omega_{j l^{\prime} m_{j}}$ are the spherical spinors, $f(r), g(r)$ are a radial functions and $l=j \pm 1, l^{\prime}=2 j-l$

Knowing that $\Omega_{j l m_{j}}$ depend on the spherical har-

where

$$
\int_{\theta=0 \phi=0}^{\pi} \int_{\phi=0}^{2 \pi} Y_{l^{\prime}}^{m^{\prime *}}(\theta, \phi) Y_{l}^{m^{*}}(\theta, \phi) \cos \theta \sin \theta \mathrm{d} \theta \mathrm{d} \phi=\left\{\begin{array}{c}
\sqrt{\frac{(l+m+1)(l-m+1)}{(2 l+1)(2 l+3)}} \text { for } l^{\prime}=l+1 \text { and } m^{\prime}=m \\
\sqrt{\frac{(l+m)(l-m)}{(2 l+1)(2 l-1)}} \quad \text { for } l^{\prime}=l-1 \text { and } m^{\prime}=m \\
0 \text { otherwise }
\end{array}\right.
$$

Let us compute first the matrix element $C_{1}(t)$ which can be written as:

$$
C_{1}(\mathrm{t})=\iint \mathrm{d} \boldsymbol{r} \mathrm{d} \boldsymbol{r}^{\prime} \phi_{2 \frac{3}{2} \frac{3}{2}}(\boldsymbol{r}) \phi_{2 \frac{3}{2} \frac{3}{2}}^{*}\left(\boldsymbol{r}^{\prime}\right)\left\{K_{a}\left(\boldsymbol{r}, t ; \boldsymbol{r}^{\prime}, 0\right)\right\}
$$

Replacing the propagator by its expression, integrating over $\boldsymbol{r}$ and $\boldsymbol{r}^{\prime}$, and using the orthogonality of the wave functions we get :

$$
\begin{aligned}
\mathrm{C}_{1}(\mathrm{t})= & \sum_{\alpha_{2}, \alpha_{3} \cdots \alpha_{k}} \sum_{k=0}^{\infty}\left(\frac{\mathrm{i}}{\hbar}\right)^{k} \frac{1}{k !} \int_{0}^{t} \mathrm{~d} \tau_{1} \cdots \int_{0}^{t} \mathrm{~d} \tau_{k} \int \mathrm{d} \boldsymbol{r}_{1} \\
& \cdots \int \mathrm{d} \boldsymbol{r}_{k}\left(\mathrm{e} \boldsymbol{E}_{1} \boldsymbol{r}\right) \cdots\left(\mathrm{e} \boldsymbol{E}_{k} \boldsymbol{r}_{k}\right) \phi_{2 \frac{3}{2}}^{*}\left(\boldsymbol{r}_{k}\right) \phi_{\alpha_{k}}\left(\boldsymbol{r}_{k}\right) \\
& \cdots \phi_{\alpha_{2}}\left(\boldsymbol{r}_{1}\right) \phi_{2 \frac{3}{2} \frac{3}{2}}^{*}\left(\boldsymbol{r}_{1}\right) \exp \left[\frac{\mathrm{i}}{\hbar} \epsilon_{2 \frac{3}{2}}(t-0)\right]
\end{aligned}
$$

Let us examine the structure of the first terms in this

expansion. Calling $U_{1}^{k}$ the successive terms in the sum over k in Equation (14), we can write the first terms as:

- term $k=0: U_{1}^{k=0}=1$

- term $k=1$ :

$$
U_{1}^{k=1}=\frac{\mathrm{ie}}{\hbar} \int_{0}^{t} \mathrm{~d} \tau_{1}\left[\boldsymbol{E}\left(\int \mathrm{d} \boldsymbol{r}_{1} \phi_{2 \frac{3}{2} \frac{3}{2}}\left(\boldsymbol{r}_{1}\right) \boldsymbol{r}_{1} \phi_{2 \frac{3}{2} \frac{3}{2}}^{*}\left(\boldsymbol{r}_{1}\right)\right)\right]=0
$$

where we have used the selection rule for the total moment $j$.

$$
\begin{aligned}
\text { - term } & k=2: \\
U_{1}^{k=2}= & \frac{1}{2}\left(\frac{\mathrm{ie}}{\hbar}\right)^{2} \int_{0}^{t} \mathrm{~d} \tau_{1} \int_{0}^{t} \mathrm{~d} \tau_{2} \cdot \sum_{\alpha_{2}}^{\infty}\left[\boldsymbol{E}\left(\int \mathrm{d} \boldsymbol{r}_{1} \phi_{2 \frac{33}{2} \frac{}{2}}\left(\boldsymbol{r}_{1}\right) \boldsymbol{r}_{1} \phi_{\alpha_{2}}^{*}\left(\boldsymbol{r}_{1}\right)\right)\right] \\
\ldots & {\left[\boldsymbol{E}\left(\int \mathrm{d} \boldsymbol{r} \phi_{\alpha_{2}}^{*}\left(\boldsymbol{r}_{2}\right) \boldsymbol{r}_{2} \phi_{2 \frac{3}{2} \frac{3}{2}}\left(\boldsymbol{r}_{2}\right)\right)\right] }
\end{aligned}
$$


In this formula, neglecting the coupling effect of the states $\left|n \neq 2, j, m_{j}\right\rangle$ with the state $\mid 2, \frac{3}{2}, \frac{1}{2}$, we obtain that:

$$
\begin{aligned}
& \left.\left[\boldsymbol{E}\left(\int \mathrm{d} \boldsymbol{r}_{1} \phi_{2 \frac{3}{2} \frac{3}{2}}\left(\boldsymbol{r}_{1}\right) \boldsymbol{r}_{1} \phi_{\alpha_{2}}^{*}\left(\boldsymbol{r}_{1}\right)\right)\right]\right\rangle \\
& {\left[\boldsymbol{E}\left(\int \mathrm{d} \boldsymbol{r} \phi_{\alpha_{2}}^{*}(\boldsymbol{r}) \boldsymbol{r} \phi_{2 \frac{3}{2} \frac{3}{2}}(\boldsymbol{r})\right)\right]=0}
\end{aligned}
$$

By using the properties of the spherical harmonics and the selection rules for the different terms of order $k$, the result of this component is :

$$
C_{1}(t)=C_{4}(t)=1
$$

If we proceed similarly for the other components: $C_{i}(t)(\mathrm{i}=2,3,5,6)$, we show that the odd terms of $k$ vanish, and only even terms contribute, and their results may be written as:

$$
\begin{aligned}
& C_{2}(t)=C_{5}(t)=\cos \left(\frac{D_{+}}{\hbar} \sqrt{h(t)}\right) \\
& C_{3}(t)=C_{6}(t)=\cos \left(\frac{D_{-}}{\hbar} \sqrt{h(t)}\right)
\end{aligned}
$$

where

$$
D_{ \pm}=\left|\mathrm{e} \int \mathrm{d} \boldsymbol{r}_{1} \phi_{2, \frac{3}{2}, \pm \frac{1}{2}}(\boldsymbol{r}) \boldsymbol{r} \phi_{2, \frac{1}{2}, \pm \frac{1}{2}}^{*}(\boldsymbol{r})\right|
$$

Summarizing (19) and (20), we can write the formula (5) for the dipolar auto-correlation function $C(t)$ as [11]:

$$
\begin{aligned}
C(t)= & \frac{1}{4}\left|2 \frac{3}{2}\right| \mathrm{d}^{1}\left|1 \frac{1}{2}\right|^{2} \exp \left(\Phi_{e}^{1} t+\frac{\mathrm{i}}{\hbar}\left(\epsilon_{2,3 / 2}-\epsilon_{1,1 / 2}\right) t\right) \\
& \cdot\left(2+\cos \left(\frac{D_{+}}{\hbar} \sqrt{h(t)}\right)+\cos \left(\frac{D_{-}}{\hbar} \sqrt{h(t)}\right)\right) \\
& +\frac{1}{2}\left|2 \frac{1}{2}\right| \mathrm{d}^{1}\left|1 \frac{1}{2}\right|^{2} \exp \left(\Phi_{e}^{2} t\right. \\
& \left.+\frac{\mathrm{i}}{\hbar}\left(\epsilon_{2,1 / 2}-\epsilon_{1,1 / 2}\right) t\right)\left(\cos \left(\frac{D_{+}}{\hbar} \sqrt{h(t)}\right)\right. \\
& \left.+\cos \left(\frac{D_{-}}{\hbar} \sqrt{h(t)}\right)\right)
\end{aligned}
$$

where

$$
h(t)=\int_{0}^{t} \mathrm{~d} \tau \int_{0}^{t} \mathrm{~d} \tau^{\prime}\left\{E(\tau) E\left(\tau^{\prime}\right)\right\}=\frac{1}{3} \int_{0}^{t} \mathrm{~d} \tau \int_{0}^{t} \mathrm{~d} \tau^{\prime}\left\{\boldsymbol{E}(\tau) \boldsymbol{E}\left(\tau^{\prime}\right)\right\}
$$

$\Phi_{e}^{1}$ and $\Phi_{e}^{2}$ are the electronic collision operators relative to $\left(2 p_{3 / 2} \rightarrow 1 s_{1 / 2}\right)$ and $\left(2 p_{1 / 2} \rightarrow 1 s_{1 / 2}\right)$ transi- tions respectively, and the symbol $\{\ldots\}$ means as said earlier the statistical average over the ionic perturbers.

Making the integral over $\tau^{\prime}$ in (22), leads to express $h(t)$ as a function of the time microfield auto-correlation function $C_{E E}(t)$. The latter represents, as known, the dynamical effect of the electric microfield on the emitters radiative properties :

$$
\begin{aligned}
h(t) & =\frac{2 t}{3} \int_{0}^{t} \mathrm{~d} \tau\left(1-\frac{\tau}{t}\right)\{\boldsymbol{E}(\tau) \boldsymbol{E}(0)\} \\
& =\frac{2 E^{2} t}{3 \omega_{p}} \int_{0}^{t} \mathrm{~d} \tau\left(1-\frac{\tau}{t}\right) C_{E E}(\tau)
\end{aligned}
$$

where $t$ means the time in the inverse of the electronic plasma frequency unit $\left(\omega_{p}=\sqrt{\frac{4 \pi N_{e} \mathrm{e}^{2}}{m_{e}}}\right)$.

Equation (21) gives the time dipolar autocorrelation function regardless of the nature of charged particles, we can apply it according to different interests, either to ions or to electrons.

\section{Conclusions}

Using the path integral formalism, we derive an expression for the Lyman alpha line shape retaining the fine structure and the effect of ion dynamics. Our main approximation is a reduction to pair correlation functions of a cluster expansion in the electric microfield.

This allows to sum all the terms appearing in the standard perturbative solution in the path integral point of view, and to express the time dipolar autocorrelation function in a compact expression involving the electric field autocorrelation function. In particular, we would like to use the ability of the path integral point of view for the description of a full quantum emitter-perturber interaction. Interesting applications of a full quantum approach exist in high temperature plasmas such as found in fusion devices, for a modelling of the emission of multicharged emitters perturbed by electrons.

\section{REFERENCES}

[1] H. R. Griem, M. Blaha and P. Kepple, "Stark-Profile Calculations for Resonance Lines of Heliumlike Argon in Dense Plasmas," Physical Review A, Vol. 41, 1990, pp. $5600-5609$.

[2] M. Baranger, "Atomic and Molecular Processes," Academic Press Inc., New York, 1962.

[3] M. Baranger, "Simplified Quantum-Mechanical Theory of Pressure Broadening," Physical Review, Vol. 111, No. 2, 1958, pp. 481-491. doi:10.1103/PhysRev.111.481

[4] A. C. Kolb and H. R. Griem, "Theory of Line Broadening in Multiplet Spectra," Physical Review, Vol. 111, 1958, pp. 514-521. 
[5] R. Feynman and A. R. Hibbs, "Quantum Mechanics and Path Integrals," McGraw- Hill, New York, 1965.

[6] H. Kleinert, "Path Integrals in Quantum Mechanics Statistics and Polymer Physics," World Scientific, Singapore, 1990.

[7] Is. Chihi, M. T. Meftah and H, Kleinert, "Path Integral Approach in the Plasma Radiation Theory," Journal of Plasma Physics, Vol. 70, 2004, pp. 553-559. doi:10.1017/S0022377803002794

[8] H. Bouguettaia, Is. Chihi, K. Chenini, M. T. Meftah, F. Khelfaoui and R. Stamm, "Application of Path Integral Formalism in Spectral Line Broadening: Lyman- $\alpha$ in Hy- drogenic Plasma," Journal of Quantitative Spectroscopy and Radiative Transfer, Vol. 94, No. 3-4, 2005, pp. 335346. doi:10.1016/j.jqsrt.2004.09.015

[9] H. R. Griem, "Spectral Line Broadening by Plasma," McGraw-Hill, New York, 1964.

[10] L. Landau and E. Lifchitz, "Théorie Quantique Relativiste," MIR, Moscou, 1972.

[11] N. Bedida, M. T. Meftah, D. Boland and R. Stamm, "Path Integral Formalism for Spectral Line Shape in Plasmas," Proceedings of the 19th International Conference on Spectral Line Shapes, Valladolid, 15-20 June 2008, pp. 100101. 